International Journal of Pure and Applied Mathematics

Volume 88 No. $4 \quad 2013,465-467$

ISSN: 1311-8080 (printed version); ISSN: 1314-3395 (on-line version)

url: http://www.ijpam.eu

doi: http://dx.doi.org/10.12732/ijpam.v88i4.1

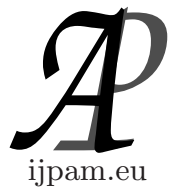

\title{
ON THE REAL VALUES OF CERTAIN \\ PARTIAL FRACTION SUMS
}

\author{
Mahfooz Alam \\ Department of Mathematics \\ King Saud University \\ P.O. Box 2455, Riyadh 11451, SAUDI ARABIA
}

\begin{abstract}
The concept of polar composite polynomials was introduced by Zaheer and Alam [1] and was studied in quite a detail in the same paper. Some further applications were obtained by the authors in their subsequent articles $[2,3]$. In the present paper the author applies the results of $[1]$ to find the real values of certain partial fraction sums.
\end{abstract}

AMS Subject Classification: 30C10, 30C15

Key Words: Join of two non-empty sets, convex hull

\section{Introduction}

We know that the non-trivial generalized circular regions of $\mathbb{C}_{w}$ (the projective field of the field $\mathbb{C}$ of complex numbers found by adjoining to $\mathbb{C}$, the scalar infinity $w$ ) are the open interior or exterior of circles or the open half-planes with a connected subset (possibly empty) of their boundary adjoined. Also, the join $\mathcal{J}[A, B]$ of two non-empty sets $A, B \subseteq \mathbb{C}$ is defined as $\mathcal{J}[A, B]=\cup\{[a, b]$ : $a \in A, b \in B\}$.

Received: February 15, 2013

(C) 2013 Academic Publications, Ltd. url: www.acadpubl.eu 
Now we state the following theorem which we shall use here.

Theorem 1. [1, Theorem 4.2]: Given a non-constant polynomial $f$ in $\mathbb{C}$, we define for all $\xi \in \mathbb{C}$, the rational function $Q(z)=\frac{(\xi-z) f^{\prime}(z)}{f(z)}$. If $A$ is a generalized circular region of $\mathbb{C}_{w}$ containing all the zeroes of $f$ and if $B$ is a non-empty set of $\mathbb{C}$, then for each $\xi \in B$, either throughout its domain (which happens if and only if $Z(f)=\{\xi\}$ ) or $Q(z)$ takes every positive real value (at least once and at most $n$ times) in the join $\mathcal{J}[A, B]$ and takes no positive value outside $\mathcal{J}[A, B]$.

Since convex sets are essentially finite or infinite intersections of certain generalized half-planes, the final analogous formulation of Theorem 1 above for the case when $A$ is any convex set not necessarily a generalized circular region has been obtained (cf. [1, Theorem 4.4]).

Theorem 2. In the notations of Theorem 1, if $A$ is any convex set such that all the zeroes of $f$ are contained in $A$, then $Q(z) \equiv-n$ throughout its domain in the case $Z(f)=\{\xi\}$ or $Q(z)$ takes every positive value (at least once and at most $n$ times $)$ in the convex hull $\mathcal{H}(A \cup\{\xi\})$ and no positive value outside it.

Here, we show as to how the above results can be applied in deriving results concerning the real values of certain partial fraction sums. In fact, we prove the following:

Theorem 3. Given complex numbers $\xi, a_{1}, \ldots, a_{n}$, let us define

$$
R(z)=\Sigma_{j=1}^{n} \frac{\xi-a_{j}}{z-a_{j}}
$$

If $A$ is a convex set containing the points $a_{1}, \ldots, a_{n}$, then either $R(z) \equiv 0$ throughout its domain (which happens if and only if $a_{j}=\xi$ for all $j$ ) or takes every real value larger than $n$ (at least once and at most $n$ times) in the convex hull $\mathcal{H}(A \cup\{\xi\})$ and takes no such value outside it.

Proof. If we define $f(z)=\Pi_{j=1}^{n}\left(z-a_{j}\right)$, we observe that

$$
\begin{gathered}
R(z)=\Sigma_{j=1}^{n}\left\{\frac{\xi-z}{z-a_{j}}+1\right\}=(\xi-z)\left\{\Sigma_{j=1}^{n} \frac{1}{z-a_{j}}\right\}+n \\
=\frac{(\xi-z) f^{\prime}(z)}{f(z)}+n=Q(z)+n,
\end{gathered}
$$

where $Q(z)-\frac{(\xi-z) f^{\prime}(z)}{f(z)}$. Now we know $Q(z) \equiv-n$ throughout its domain (if and only if $Z(f)=\{\xi\}$ or that $Q(z)$ takes every positive value at least once 
and most of $n$ points in the complex plane. Therefore, we would have finished if we could only show that all these points lie in the convex hull $\mathcal{H}(A \cup\{\xi\})$. But this has been shown in the proof of Theorem 2. Since $A$ is a convex set and $f$ is a polynomial of degree $n$ with its zeroes in $A$, our theorem follows.

We state the following consequence of Theorem 3 :

Corollary 1. Under the notations of Theorem 3 , if $a_{1}, \ldots, a_{n}$ are all real, then for each $\xi$ lying in the upper (respectively. lower) closed half-plane, the function $R(z)$, if not identically zero, takes every real value larger than $n$ in the upper (resp. lower) closed half-plane and at no point outside it.

Remark 1. In fact, Theorem 3 is the reformulations of Theorem 2 in terms of partial fraction sums. One could also obtain a similar reformulation of Theorem 1. The zeroes of $R(z)$ can be interpreted in various ways from the stand point of Physics, Geometry and Theory of Functions. Hence some interesting applications in certain physical phenomena such as gravitational and electromagnetic attraction and two-dimensional fluid motion can be found.

\section{Acknowledgments}

The author is thankful to Prof. Neyamat Zaheer for his valuable comments.

\section{References}

[1] N. Zaheer, M. Alam, Zeroes of polar-composite polynomials in algebraically closed fields, Proc. London Math. Soc., 40, No. 3 (1980), 527-552.

[2] N. Zaheer, M. Alam, Some applications of the theory of polar-composite polynomials, J. London Math. Soc., 22, No. 2 (1980), 403-410.

[3] N. Zaheer, M. Alam, Certain applications of the theory of polar-composite polynomial, Proc. Amer. Math. Soc., 85, No. 3 (1982), 383-388. 
